\title{
Effects of Latent Heating on Atmospheres of Brown Dwarfs and Directly Imaged Planets
}

\author{
Xianyu Tan and Adam P. Showman \\ Department of Planetary Sciences and Lunar and Planetary Laboratory, University of Arizona, 1629 University Boulevard, Tucson, AZ 85721, USA; \\ xianyut@1pl.arizona.edu \\ Received 2016 June 15; revised 2016 December 8; accepted 2016 December 9; published 2017 January 30
}

\begin{abstract}
The growing number of observations of brown dwarfs (BDs) has provided evidence for strong atmospheric circulation on these objects. Directly imaged planets share similar observations and can be viewed as low-gravity versions of BDs. Vigorous condensate cycles of chemical species in their atmospheres are inferred by observations and theoretical studies, and latent heating associated with condensation is expected to be important in shaping atmospheric circulation and influencing cloud patchiness. We present a qualitative description of the mechanisms by which condensational latent heating influences circulation, and then illustrate them using an idealized general circulation model that includes a condensation cycle of silicates with latent heating and molecular weight effect due to the rainout of the condensate. Simulations with conditions appropriate for typical $T$ dwarfs exhibit the development of localized storms and east-west jets. The storms are spatially inhomogeneous, evolving on a timescale of hours to days and extending vertically from the condensation level to the tropopause. The fractional area of the BD covered by active storms is small. Based on a simple analytic model, we quantitatively explain the area fraction of moist plumes and show its dependence on the radiative timescale and convective available potential energy (CAPE). We predict that if latent heating dominates cloud formation processes, the fractional coverage area of clouds decreases as the spectral type goes through the $\mathrm{L} / \mathrm{T}$ transition from high to lower effective temperature. This is a natural consequence of the variation of the radiative timescale and CAPE with the spectral type.
\end{abstract}

Key words: brown dwarfs - hydrodynamics - methods: numerical - planets and satellites: atmospheres planets and satellites: gaseous planets

\section{Introduction}

Observations of brown dwarfs (BDs) have shown increasing evidence of vigorous circulation in the BDs' atmospheres (Showman \& Kaspi 2013). This evidence includes near-infrared brightness variability (Artigau et al. 2009; Radigan et al. 2012, 2014; Apai et al. 2013; Buenzli et al. 2014, 2015; Wilson et al. 2014; Metchev et al. 2015; Yang et al. 2015, 2016; Cushing et al. 2016), chemical disequilibrium (Fegley \& Lodders 1996; Saumon et al. 2006, 2007; Hubeny \& Burrows 2007; Stephens et al. 2009; Visscher \& Moses 2011; Zahnle \& Marley 2014), and surface patchiness (Crossfield et al. 2014). Cloud disruption has been proposed to help explain properties of the $\mathrm{L} / \mathrm{T}$ transition (Ackerman \& Marley 2001; Burgasser et al. 2002; Marley et al. 2010), and such patchiness is also likely to be responsible for the near-infrared brightness variability (Marley \& Robinson 2015). However, the mechanism responsible for cloud disruption is yet unclear. Atmospheric circulation is expected to play a crucial role in controlling the cloud coverage fraction, but the details remain poorly understood.

A handful of directly imaged extrasolar giant planets (EGPs) exhibit similarities with BDs: near-infrared colors, inference of dust and clouds, chemical disequilibrium in their atmospheres, and a fast spin (Hinz et al. 2010; Barman et al. 2011a, 2011b; Marley et al. 2012; Oppenheimer et al. 2013; Ingraham et al. 2014; Skemer et al. 2014; Snellen et al. 2014; Macintosh et al. 2015; Wagner et al. 2016). Near-IR brightness variability has also recently been observed on directly imaged EGPs (Biller et al. 2015; Zhou et al. 2016). From a meteorological point of view, directly imaged EGPs resemble low-gravity versions of BDs, and their atmospheric dynamical regime is characterized by fast rotation, vigorous convection, and negligible external heating.
Motivated by observations, several studies have explored the atmospheric dynamics of ultracool objects (compared to stars). Local two-dimensional hydrodynamic simulations by Freytag et al. (2010) showed that interactions between the convective interior and the stratified layer can generate gravity waves that propagate upward, and the breaking of these waves causes vertical mixing that leads to small-scale cloud patchiness. Showman \& Kaspi (2013) presented the first global model of $\mathrm{BD}$ dynamics for the convective interior and showed that largescale convection is dominated by fast rotation. Using an analytic theory, they proposed that atmospheric circulation can be driven by atmospheric waves in the stably stratified upper atmosphere. Using a two-layer shallow-water model, Zhang \& Showman (2014) showed that weak radiative dissipation and strong forcing favor large-scale zonal jets for BDs, whereas strong dissipation and weak forcing favor transient eddies and quasi-isotropic turbulence. Despite these studies, no global model that includes condensate cycles and clouds has yet been published for BDs. Clouds play a significant role in sculpting the temperature structure, spectra, and brightness variations of BDs (see the recent reviews of Marley \& Robinson 2015 and Helling \& Casewell 2014). There is a pressing need to couple condensation cycles and clouds to global models to study how the circulation controls global cloud patchiness and, in turn, how the condensation cycle affects the circulation.

In this paper, we propose the importance of latent heating in the atmospheric circulation and cloud patchiness of BDs by using an idealized general circulation model (GCM) that includes a condensation cycle of silicate vapor. Latent heating is of paramount importance in Earth's atmosphere (Emanuel 1994). For giant planets in our solar system whose atmospheres are likely analogous to BDs', a long history of studies have shown the importance of latent heating in driving their atmospheric 
circulation (Barcilon \& Gierasch 1970; Gierasch 1976; Gierasch et al. 2000; Ingersoll et al. 2000; Lian \& Showman 2010). Lian \& Showman (2010) demonstrated that large-scale latent heating from the condensation of water can drive patterns of zonal (eastwest) jet streams that resemble those on all four giant planets of the solar system: numerous zonal jets off the equator and a strong prograde equatorial jet on Jupiter and Saturn and a three-jet pattern including retrograde equatorial flow and high-latitude prograde flow on Uranus and Neptune. Such models also exhibit episodic storms that qualitatively resemble those observed on Jupiter and Saturn. For BDs, the evidence for patchy clouds' effect on brightness variability and the L/T transition itself also suggests a strong role for an active condensate cycle, and latent heating may be similarly important for the atmospheric circulation of BDs and directly imaged EGPs. Because temperature perturbations associated with (dry) convection at condensable pressure levels are generally small, the latent heating that accompanies the condensation of relevant chemical species can dominate the buoyancy in layers where condensation occurs.

The main point of this paper is to illustrate how latent heating modifies circulation and influences cloud patchiness in the simplest possible context, so we intentionally exclude clouds, radiative transfer, and detailed microphysics to allow a simpler environment in which to clarify the dynamical processes that are at play. Cloud microphysics processes are highly complex (Rossow 1978), and significant prior work on the cloud microphysics issue (see a review by Helling \& Casewell 2014) as well as parameterized cloud models (Allard et al. 2001; Ackerman \& Marley 2001; Tsuji 2002; Cooper et al. 2003; Barman et al. 2011a) has been done for ultracool atmospheres. We are well aware of the important feedback of clouds to atmospheres and leave it for future efforts. Also, to simulate vigorous convection and the dynamics in the radiative-convective boundary caused by convective perturbation, one needs a model that can properly treat both the convective interior and the overlying stably stratified layer. Therefore, we do not expect our current simulations to resemble the true atmospheres of BDs and directly imaged EGPs.

The paper is organized as follows. We start out in Section 2 by describing several important effects of latent heating on the atmosphere; in Section 3, we briefly introduce the idealized model that we use to illustrate the mechanisms described in Section 2; in Section 4, we show the result of our simulations; and in Section 5, we discuss our results and their implications for observations and draw conclusions.

\section{Effects of Latent Heating on Atmospheres}

\subsection{Conditional Instability}

Most atmospheres of planets and ultracool BDs have constituents that can condense. Due to atmospheric motion and diabatic heating/cooling, air parcels containing condensable species can undergo a change of temperature and pressure, which leads to condensation. The latent heating/ cooling due to condensation/evaporation has important effects on the stability of atmospheres, which we summarize here; a more detailed discussion can be found, e.g., in Chapter 7 of Salby (2012). For simplicity, we begin our discussion assuming the molecular weight is constant, but we return to this issue in a later subsection.
It is well known that a rapidly ascending or descending dry air parcel follows a dry adiabatic lapse rate

$$
\frac{d \ln T}{d \ln p}=\frac{R}{c_{p}},
$$

where $T$ is the temperature, $p$ is the pressure, $R$ is the specific gas constant, and $c_{p}$ is the specific heat capacity of the dry atmosphere. Similarly, a saturated air parcel mixed with condensable and noncondensable gases follows a moist adiabatic lapse rate ${ }^{1}$

$$
\frac{d \ln T}{d \ln p}=\frac{R_{u}+\frac{L_{m} \xi}{T}}{c_{p}+\frac{L_{m}^{2} \xi}{R_{u} T^{2}}},
$$

where $L_{m}$ is the latent heat per mole, $c_{p}$ is the specific heat capacity per mole for the mixture, $R_{u}$ is the universal gas constant, and $\xi=p_{\text {cond }} / p_{d}$ is the molar mixing ratio of the condensable gas to the dry gas. Under the normal conditions of most atmospheres, the dry adiabat is larger than the moist adiabat as long as $L_{m}>c_{p} \mathrm{~T}$. In the presence of two adiabats, the atmosphere can have different stability criteria. If the atmospheric lapse rate $\frac{d \ln T}{d \ln p}$ is higher than the dry adiabatic lapse rate $R / c_{p}$, the atmosphere will be absolutely unstable; if the lapse rate is lower than the moist adiabatic lapse rate, the atmosphere will be absolutely stable; and if the lapse rate is in between the dry and moist adiabatic lapse rates, the atmosphere is stable against dry convection but unstable to moist convection, which is referred to as conditional instability. Examples include the tropospheres of the Earth, Titan, and probably Jupiter and Saturn.

How does an air parcel behave in a conditional unstable and unsaturated atmosphere? This is schematically illustrated in Figure 1: starting from an arbitrary level below the condensation level, the ascending air parcel follows a dry adiabat until its relative humidity reaches $100 \%$, and reaches the lifting condensation level. Afterward it follows a moist adiabat, and then at some point it reaches the level of free convection (LFC), where it has a lower density than the environment and becomes positively buoyant. The air parcel can then freely convect to the top of a cumulus storm, where its buoyancy diminishes and it stops ascending. In reality, because the atmospheric lapse rate may be stable to dry convection, some external lifting mechanism is needed for the air parcel to reach the LFC, and this is why storms do not occur everywhere and in every moment in Earth's tropics even though the atmosphere is conditionally unstable. The amount of energy required to reach the LFC is referred to as convective inhibition (CIN). To initiate moist convection, either strong initial diabatic heating (e.g., in the case of Earth, heating of the surface by sunlight) or kinetic energy (e.g., forced lifting by atmospheric waves or other large-scale motion) is needed for the parcel to overcome CIN. CIN can act to limit the frequency of moist convection and preserve high convective available potential energy (CAPE), which can be essential for the development of deep moist convection.

\footnotetext{
1 In deriving this formula, we used the Clausius-Clapeyron equation for the saturation vapor pressure of the condensable species, assuming an ideal gas equation of state and that the condensate density is much greater than the gas density. This formula is applicable for the full range of $\xi$ and not limited to the assumption of a low mixing ratio of condensable gas.
} 


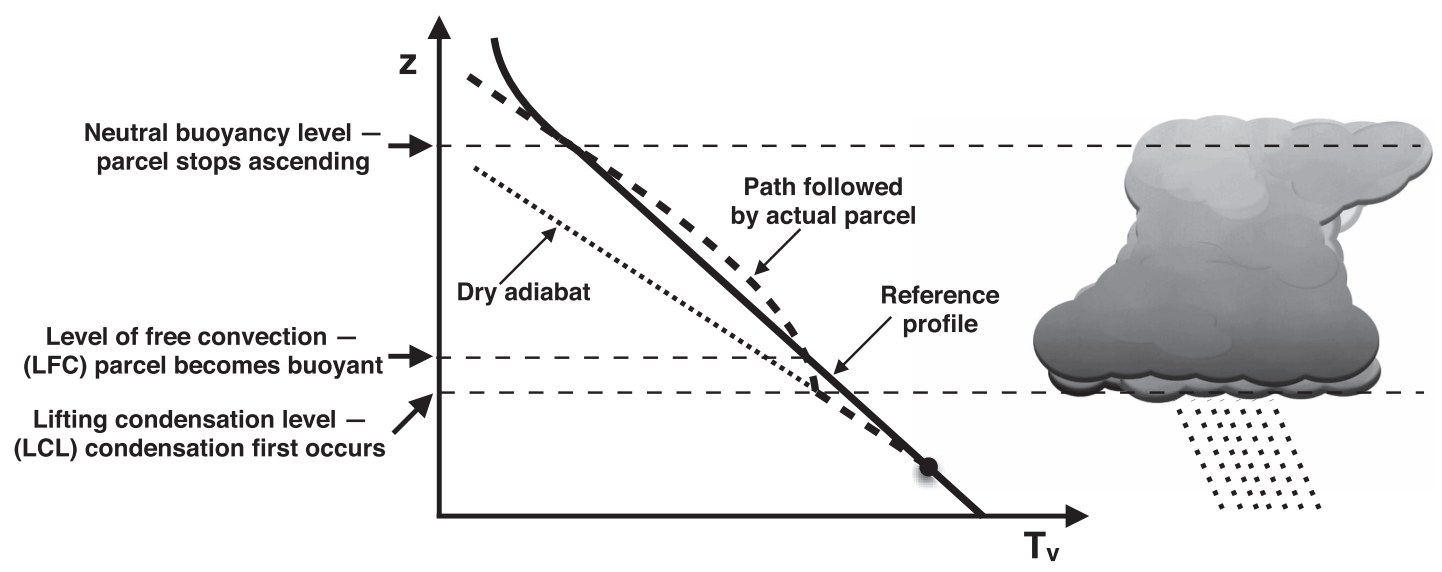

Figure 1. A schematic plot showing how an air parcel behaves in a conditionally unstable atmosphere. The solid line is the background reference temperature profile, the dashed line is the path followed by an actual air parcel initiated below the lifting condensation level, and the dotted line indicates the dry adiabatic path if the air parcel is dry. The horizontal axis is the temperature (or, if molecular weight effects are important, the virtual temperature), and the vertical axis is the height within the atmosphere.

One necessary condition for conditional instability is stratification to dry convection in the troposphere. One mechanism to produce tropospheric stratification is latent heating and moist convection. As illustrated in Figure 1, the rising air that follows a moist adiabat in storms carries a higher entropy than where it is initiated. Mass continuity implies that the high-entropy surrounding atmosphere at the top of the storm must subside. During subsidence, air continues to lose its entropy to space via IR radiation. Air closer to the ground has been subsiding for longer-and thus exhibits lower entropythan air aloft. As a result, the entropy increases with height in the background temperature profile, which implies that the background temperature profile is stable to dry convection. On the other hand, the background temperature is lower than the temperature of the moist adiabat, allowing moist instability. For giant planets, tropospheric stratification has been inferred from observations for Jupiter (Flasar \& Gierasch 1986; Magalhães et al. 2002; Reuter et al. 2007), and it has been demonstrated by numerical simulations that the stratification in Jupiter could have resulted from the latent heating of water condensation (Nakajima et al. 2000; Sugiyama et al. 2014).

\subsection{Moist Convection Controlling the Area Fraction of Moist Plumes}

Vertical velocity within the moist convecting plume is much higher than the surrounding subsidence flow, as observed in Earth's atmosphere. The high upwelling velocity is driven by the high CAPE in deep moist convection. CAPE is the amount of potential energy per unit mass available for the convection of a particular air parcel and is essentially an integration of buoyancy with respect to height during the lifting of the parcel (e.g., Emanuel 1994, Chapter 6). In contrast to the buoyant ascending convective plumes, the subsiding air is stratified and not convecting, but it can gradually subside as described in Section 2.1. Because the radiative cooling timescale is generally long, the subsidence is generally slow. The asymmetry of the upwelling and downwelling vertical velocity results in a small area fraction of moist ascending plumes by the requirement of mass continuity. The area covered by clouds may not closely follow the area of moist plumes because cloud particles are spread by the wind field near the cloud top, but this argument for the moist plume area fraction can qualitatively explain the origin of the patchiness of cumulus clouds (Lunine \& Hunten 1987). For BDs, patchy clouds deduced from nearIR brightness variability may qualitatively be explained by this mechanism.

\subsection{Molecular Weight Effect}

In the atmospheres of gaseous giant planets and BDs, condensable species generally have a much greater molecular weight than the dominant dry constituent $\mathrm{H}_{2}$. Rainout of these condensates can decrease the density of air, and this effect can play an important role in atmospheric thermal structure and dynamics. For example, Guillot (1995) presented the idea that moist convection in giant planets may be inhibited by a molecular weight effect if the mixing ratio of water is substantially higher than the solar abundance. Also, Li \& Ingersoll (2015) proposed that Saturn's 20-30 yr quasi-periodic planetary-scale storm is related to the molecular weight effect of water. In BDs and directly imaged EGPs, a local thin stably stratified layer associated with molecular weight gradients may exist right above the condensation level, due to the fact that the subsiding environmental air has experienced rainout and is thus relatively dry and has lower molecular mass. In contrast, the air below the condensation level contains significant condensable vapor and has higher molecular mass. Therefore, a sharp gradient in molecular mass naturally exists near the condensation level, where molecular mass decreases with increasing altitude. This produces stratification to both dry and moist convection, contributing to CIN. High CIN can suppress moist convection within (and above) the stable layer, leaving the atmosphere to gradually cool off toward its radiative equilibrium by thermal radiation. The radiative equilibrium temperature profile in the troposphere (for instance, on Earth and Jupiter) usually has a temperature lapse rate $d \ln T / d \ln p$ higher than the moist adiabatic lapse rate. Thus, the existence of CIN can help contribute to the accumulation of CAPE. This phenomenon has also been shown by simulation on Jupiter (Nakajima et al. 2000). By its contribution to the accumulation of CAPE, the stratification from the molecular weight effect also helps to control the moist plume fraction via the mechanism discussed above. 


\subsection{Large-scale Latent Heating on Atmospheric Dynamics}

Moist convection provides a source of small-scale eddies, which can grow into large-scale eddies via an inverse energy cascade. The interactions among these eddies and the mean flow in a rapidly rotating sphere can produce zonally banded structure and vortices (Lian \& Showman 2010; also see a review by Vasavada \& Showman 2005 for Jovian atmospheric dynamics). Latent heating can interact with the dynamics in many ways and may produce organized clouds that can lead to cloud radiative feedback to the dynamics. Temperature perturbations by latent heating on isobaric surfaces can generate a wealth of waves that propagate upward to the stratosphere, driving circulation by the dissipation and breaking of these waves (Showman \& Kaspi 2013).

\section{Model}

Here we summarize the key aspects of our model; for detailed implementation see Lian \& Showman (2010). We solve three-dimensional hydrostatic primitive equations using an atmospheric GCM, the MITgcm (Adcroft et al. 2004; see also mitgcm.org). The horizontal momentum, hydrostatic equilibrium, continuity, thermodynamic energy, and tracer equations in pressure coordinates are, respectively,

$$
\begin{gathered}
\frac{d \boldsymbol{v}}{d t}+f \hat{k} \times \boldsymbol{v}+\nabla_{p} \Phi=0 \\
\frac{\partial \Phi}{\partial p}=-\frac{1}{\rho} \\
\nabla_{p} \cdot \boldsymbol{v}+\frac{\partial \omega}{\partial p}=0 \\
\frac{d \theta}{d t}=-\frac{\theta-\theta_{\text {ref }}}{\tau_{\text {rad }}}+\frac{L \theta}{c_{p} T}\left(\delta \frac{q-q_{s}}{\tau_{\text {cond }}}\right), \\
\frac{d q}{d t}=-\delta \frac{q-q_{s}}{\tau_{\text {cond }}}+Q_{\text {deep }},
\end{gathered}
$$

where $v$ is the horizontal velocity vector on isobars, $\omega=d p / d t$ is the vertical velocity in pressure coordinates, $f=2 \Omega \sin \phi$ is the Coriolis parameter (here $\phi$ is the latitude and $\Omega$ is the planetary rotation rate), $\Phi$ is the geopotential, $\hat{k}$ is the local unit vector in the vertical direction, $\rho$ is the density, $\nabla_{\mathrm{p}}$ is the horizontal gradient in pressure coordinates, $d / d t=\partial / \partial t+$ $\boldsymbol{v} \cdot \nabla_{p}+\omega \partial / \partial p$ is the material derivative, $\theta=T\left(\frac{p_{0}}{p}\right)^{R / c_{p}}$ is the potential temperature, $p_{0}=1$ bar is a reference pressure, $\theta_{\text {ref }}$ is the equilibrium potential temperature profile, $\tau_{\text {rad }}$ is the radiative timescale, and $L$ is the latent heat per mass for the condensate. The ideal gas law is assumed for the equation of state for the atmosphere.

The hydrostatic assumption used in the standard primitive equations solved in our model is a good approximation for large-scale flows in stratified atmospheres with a high ratio of horizontal scale to vertical scale (e.g., see Vallis 2006, Chapter 2 ). In the atmospheres of BDs, the expected horizontal length scale of large-scale dynamics is $10^{6}-10^{7} \mathrm{~m}$, while the pressure scale height is $10^{3}-10^{4} \mathrm{~m}$. The aspect ratio of the atmosphere is on the order of $10^{2}-10^{3}$, which is sufficient for the hydrostatic approximation to hold.

The tracer $q$ is the mass mixing ratio of condensable vapor to dry air, and $q_{s}$ is the local saturation vapor mass mixing ratio, which is determined by the saturation pressure function for specific condensable species. The "on-off switch" function $\delta$ controls the condensation: when $q>q_{s}, \delta=1$ and vapor condenses over a characteristic timescale $\tau_{\text {cond }}$ generally taken as $10^{3} \mathrm{~s}$, representative of a typical convective time; when $q \leqslant q_{s}, \delta=0$. Latent heating is immediately applied in the thermodynamic equation (Equation (6)) once condensation occurs. For simplicity, we include only one tracer and choose enstatite vapor $\left(\mathrm{MgSiO}_{3}\right)$ to represent silicate vapor in our BD models. Silicates are one of the most abundant condensates in the atmospheres of L/T dwarfs, and their condensation levels are closer to the photospheres than those of another dominant condensate, iron, so silicates could have more influence on the photospheres of $\mathrm{L} / \mathrm{T}$ dwarfs than iron. The saturation pressure function for $\mathrm{MgSiO}_{3}$ is adopted from Ackerman \& Marley (2001). Alternative saturation pressure functions for silicates are available-for example, see Visscher et al. (2010). Our study does not aim to precisely determine where condensation occurs but rather to explore the dynamics driven by latent heating given a plausible condensation curve for a representative condensing species. The latent heat of silicates is similar in Ackerman \& Marley (2001) and Visscher et al. (2010); therefore, a detailed choice of the saturation $T-P$ profile is not essential here. The saturation pressure function reads

$$
e_{\mathrm{s}}=\exp \left(25.37-\frac{58663 \mathrm{~K}}{T}\right) \text { bar }
$$

which is shown by the dashed line in Figure 2 assuming solar abundance for the mixing ratio of the silicates. Here we assume that the condensate will be rained out immediately. The influence of the rainout of condensable vapor on air density is properly included in the hydrostatic equilibrium equation, where the density is affected by the mean molecular weight. The replenishment term $Q_{\text {deep }}$ crudely parameterizes evaporating precipitation and condensable species mixed upward from the deeper atmosphere. It takes the form $Q_{\text {deep }}=\left(q_{\text {deep }}-\right.$ $q) / \tau_{\text {rep }}$, where $q_{\text {deep }}$ is a specified abundance of condensable species in the deep atmosphere and $\tau_{\text {rep }}$ is the replenishment timescale, which is typically taken as $10^{3} \mathrm{~s}$. Both $\tau_{\text {cond }}$ and $\tau_{\text {rep }}$ are chosen to be short compared to dynamical timescales, and in this limit the dynamics should be independent of the two timescales. The $Q_{\text {deep }}$ term is applied only at levels deeper than the condensation level.

The radiation effects of the system are simplified by using the Newtonian cooling scheme (Equation (6)). For simplicity, the radiative timescale $\tau_{\text {rad }}$ is taken to be constant throughout the atmosphere. In our application, the radiative equilibrium potential temperature $\theta_{\text {ref }}$ is assumed to be spherically symmetric and is characterized by two regimes, a nearly adiabatic deeper region and an isothermal upper region, $\theta_{\text {ref }}(p)=\left[\theta_{\text {adi }}^{n}(p)+\theta_{\text {iso }}^{n}(p)\right]^{1 / n}$, where $\theta_{\text {adi }}$ represents the potential temperature of the nearly adiabatic lower layer, $\theta_{\text {iso }}$ represents that of the isothermal upper layer, and $n$ is a smoothing parameter that we here set to 15 . The equilibrium temperature profile is intended to crudely mimic the results from one-dimensional radiative-convective models, where the profile is nearly isothermal in the upper atmosphere and smoothly transitions to an adiabatic profile in the lower atmosphere (e.g., Marley et al. 2002; Burrows et al. 2006; Morley et al. 2014). Our equilibrium temperature structure is based on a gray radiative-convective calculation using the 


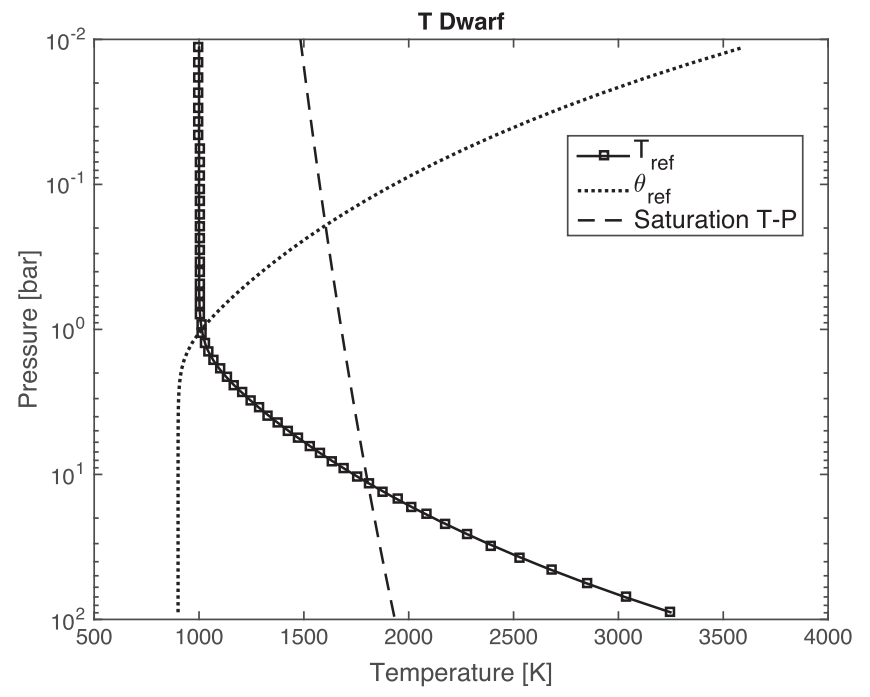

Figure 2. Equilibrium $T-P$ profile $T_{\text {ref }}$ in the model (solid line) with squares representing the model pressure levels, and the saturation $T-P$ profile (dashed line) of enstatite $\left(\mathrm{MgSiO}_{3}\right)$ for a typical $\mathrm{T}$ dwarf temperature regime assuming $1 \times$ solar abundance. The dotted line is the corresponding potential temperature $\theta_{\text {ref }}$ profile.

Rosseland mean opacity table from Freedman et al. (2014), and the radiative-convective boundary from our calculation is in good agreement with models using realistic opacities (e.g., Tsuji 2002). The deep thermal structure is generally slightly unstable rather than strictly neutral to allow dry convective motions. We parameterize the temperature structure of the adiabatic deep region by

$$
\theta_{\text {adi }}(p)=\theta_{0}+\delta \theta \log \frac{p}{p_{\text {bot }}}
$$

where $\theta_{0}$ and $\delta \theta$ are constants and $p_{\text {bot }}$ is the bottom pressure of the simulation domain. $\delta \theta$ is typically taken as $1 \mathrm{~K}$ for $1 \times$ solar cases, which is qualitatively consistent with the argument in mixing length theory but small enough to not affect the dynamics above the condensation level. Figure 2 shows the equilibrium temperature and saturation vapor $T-P$ profile for a typical $\mathrm{T}$ dwarf temperature regime, with the silicates' condensation $T-P$ curve. The dotted line is the corresponding potential temperature $\theta_{\text {ref }}$ profile, in which the adiabatic layer is characterized by nearly constant $\theta$ and the isothermal layer has increasing $\theta$ with increasing altitude.

Real moist convection involves the formation of cumulus clouds and thunderstorms on a length scale much smaller than can be resolved by most GCMs. There has been a long history of development for schemes that parameterize the effects of subgrid-scale cumulus convection on large-scale flows resolved by global models (for a review see, e.g., Emanuel \& Raymond 1993). These schemes are often complex, with concepts and parameterizations constrained by Earth's atmosphere. It is not yet clear how relevant the specific parameterization of these schemes is to the atmospheres of BDs and giant planets, so we do not include a moist convection subgrid-scale scheme in our current model. As stated in Lian \& Showman (2010), it is useful to first ascertain the effects of large-scale latent heating associated with the hydrostatic interactions of storms with the surroundings, as we pursue here.

We include a weak linear damping of velocities similar to that of Liu \& Showman (2013) at pressure larger than 50 bars to mimic the reduction of winds due to the Lorentz force and ohmic dissipation at great depths, where magnetic coupling may be important. This drag is deep and weak enough (with a timescale of 100 days at the bottom) not to affect the dynamics above the condensation level.

We solve the equations of our global model on a sphere using the cube-sphere coordinate system (Adcroft et al. 2004; Showman et al. 2009). For most of the simulations, we assume a Jupiter radius, a $5 \mathrm{hr}$ rotation period, and a $500 \mathrm{~m} \mathrm{~s}^{-2}$ surface gravity. The resolution in our nominal simulations is $\mathrm{C} 128$, which is equivalent to 0.7 per grid longitudinally and latitudinally (i.e., an approximate resolution of $512 \times 256$ in longitude and latitude). The pressure domain in our model is from 0.01 to 100 bars, and it is divided into 55 layers with finer resolution on the condensation layers, as shown in Figure 2. The horizontal and vertical resolution is adequate to resolve the Rossby deformation radius, which is the typical length scale of eddies expected on BDs, and the vapor partial pressure scale height above the condensation level, respectively. We do not include an explicit viscosity in our simulations, but a fourthorder Shapiro filter is added to the time derivative of $v$ and $\theta$ to maintain numerical stability.

\section{Results}

\subsection{A Typical T Dwarf}

We begin by describing in detail a specific representative case for a typical $\mathrm{T}$ dwarf with a radiative timescale $\tau_{\text {rad }}$ of $10^{6} \mathrm{~s}$, solar metallicity that is typical for field BDs (e.g., Leggett et al. 2010), and other parameters described in Section 3 (see also Figure 2). The spin-up time is about 1500 days for models with $\tau_{\text {rad }}=10^{6}$ and $10^{7} \mathrm{~s}$ and about 1000 days for models with $\tau_{\text {rad }}=10^{5}$ s. Figure 3 shows a snapshot of a horizontal map of temperature and zonal (east-west) velocity at 1736 Earth days of simulation time at 9.1 bars (upper row) near the condensation level. The simulation reached a statistical equilibrium state, where latent heating from the condensate cycle is statistically balanced by radiative cooling and the upward transport of condensable vapor is balanced by rainout in storms. On the temperature map (left panel), the red local regions are storms with warm upwelling moist plumes, and they evolve on a timescale of hours to (Earth) days. The upper right panel in Figure 3 shows the zonal wind map at the same pressure level, with yellow and red colors representing eastward velocity. Three eastward jets form near the equator, with maximum wind speed of about $40 \mathrm{~m} \mathrm{~s}^{-1}$. The jets are located where storms are generated, suggesting that jets are pumped by momentum transport associated with the storms. No jets form at mid-to-high latitudes, but velocity residuals manifest there, which are Rossby waves propagating northward and southward of the storm regions.

The upwelling vertical motions are strongly suppressed near the stably stratified isothermal layer at $p<2$ bars, causing large horizontal velocity divergence; as a result, the wider spreading of the upwelling hot air produces larger temperature perturbation patterns near the tropopause (lower left panel in Figure 3), similar to simulations for the Jupiter model (Lian \& Showman 2010). Because the ascending air inside storms extends vertically from the storm base to the top near the tropopause, the locations of warm regions at 3.5 bars are generally correlated to those at about 9 bars. The horizontal zonal velocity map at the tropopause exhibits multiple-jet 
Pressure $=9.1[\mathrm{bar}]$

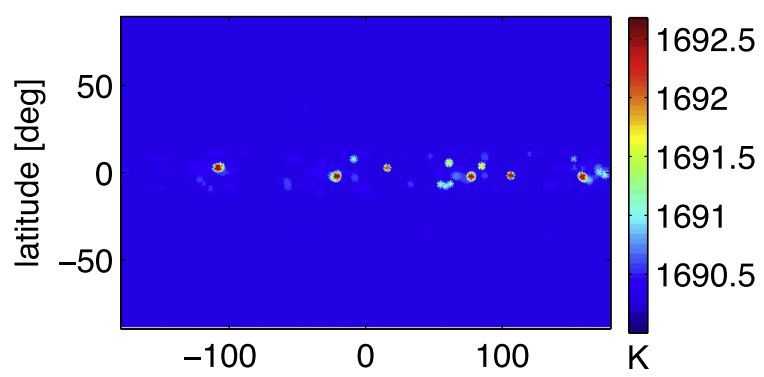

Pressure $=3.5[\mathrm{bar}]$

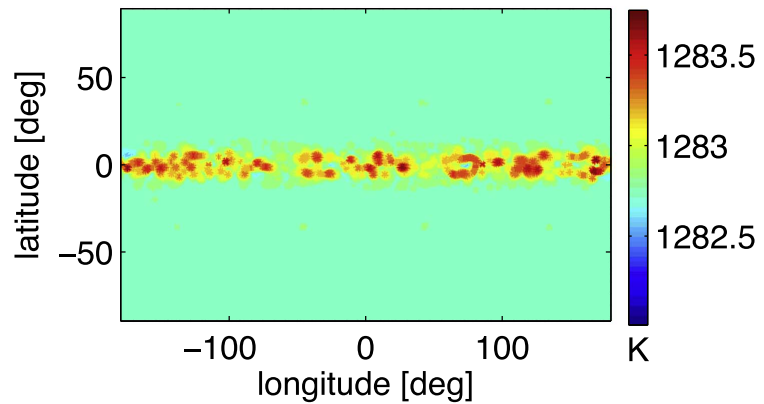

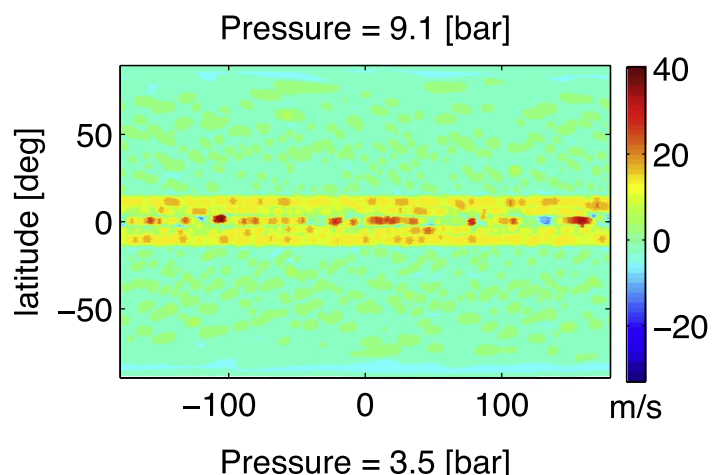

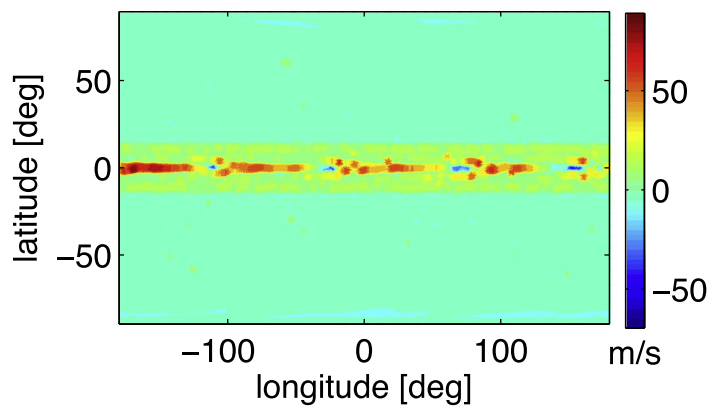

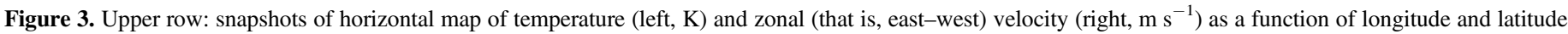

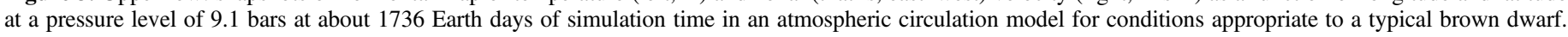
Lower row: same as the upper row but at a pressure level of 3.5 bars, near the tropopause.

configuration similar to that at 9 bars but with a higher maximum wind speed of about $90 \mathrm{~m} \mathrm{~s}^{-1}$. The larger horizontal velocity divergence near the tropopause causes more abundant turbulence and wave sources at this layer and hence stronger interactions of mean flow with turbulence and Rossby waves, generating stronger zonal flows.

Storms occur mostly near the equator-almost never in midto-high latitudes. Notice that in our model setup the equilibrium potential temperature profile $\theta_{\text {ref }}$ is independent of latitude, such that we can exclude any latitude-dependent forcing as a possible cause of the banded structure seen in our simulations. Rather, any zonal banding or latitude dependence of storms must result from the latitudinal variation of $f$ and $\beta$ (where $\beta=d f / d y$ is the gradient of the Coriolis parameter with northward distance), which are the only sources that can introduce anisotropy in our simulations (Lian \& Showman 2010).

Diagnosing the dynamical mechanism for latitude-dependent storms is difficult. Instead we offer speculation based on the fact that $f$ and $\beta$ are the only possible sources of anisotropy (and therefore of any latitude dependence). A possible reason is that the horizontal divergence of horizontal winds, $\nabla_{p} \cdot v$, tends to be smaller at mid-to-high latitudes, implying lower vertical velocities, which make it more difficult to generate and maintain storms; the suppression of storms in turn further weakens vertical velocities by limiting horizontal temperature differences, which are essential to drive horizontal divergence. There are two reasons why we expect small horizontal divergence at mid-to-high latitudes. First, winds tend to be more geostrophic (the balance between Coriolis and pressure gradient forces in the horizontal momentum Equation (3)) at higher latitudes, where the Coriolis parameter $f$ is larger, and this can lead to smaller horizontal divergence. At low latitudes, winds have larger ageostrophic components, which result in larger horizontal divergence. Thus, moist instability can be more easily triggered. The importance of rotation can be characterized by the Rossby number, $R o=U / \mathcal{L} f$, where $U$ and $\mathcal{L}$ are the characteristic horizontal wind speed and horizontal length scale, respectively. If $R o \ll 1$, the winds are nearly geostrophic. We can quantitatively estimate the latitude above which the flow tends to be geostrophic by taking $U \sim 100 \mathrm{~m} \mathrm{~s}^{-1}$ and $\mathcal{L} \sim 10^{6} \mathrm{~m}$, which are approximately the maximum relative velocity and width of a local storm, respectively, and setting $\operatorname{Ro} \sim 1$, and we have $\phi \sim 8^{\circ}$. This is qualitatively consistent with our simulations, in which storms tend to clump inside $\pm 10^{\circ}$ latitudes (Figure 3). Second, even if the flow were geostrophic, the horizontal divergence is $\nabla_{p} \cdot \boldsymbol{v} \sim \frac{v}{a \tan \phi}$, where $v$ is the meridional (north-south) velocity and $a$ is the radius of the BD. The divergence in pure geostrophic flow comes from the gradient of $f$ with respect to the latitudes. It is easy to see that even in geostrophic flow, horizontal divergence becomes smaller at higher latitudes and larger at lower latitudes. However, the argument here does not mean that there are no vertical motions at high latitudes in general situations. In fact, if one imposes an independent meridional temperature difference on the atmosphere, it is easy to generate vertical motion and overturning circulation at high latitudes (e.g., Williams 2003; Lian \& Showman 2008). The difficulty in generating high-latitude storms here is that the horizontal temperature differences that would be required for vertical motions are not independently generated but can only come from the existence of vertical motion (and the associated latent heating). This additional sensitivity allows for the suppression of storms in situations where vertical motions tend to be smaller, as at high latitudes.

Storm regions are buoyant, thereby causing ascending motion, which transports moist air upward from below. This leads to condensation and latent heating, thereby maintaining the storms themselves. Therefore, storms are spatially well correlated with the vapor mixing ratio and vertical velocities, as 


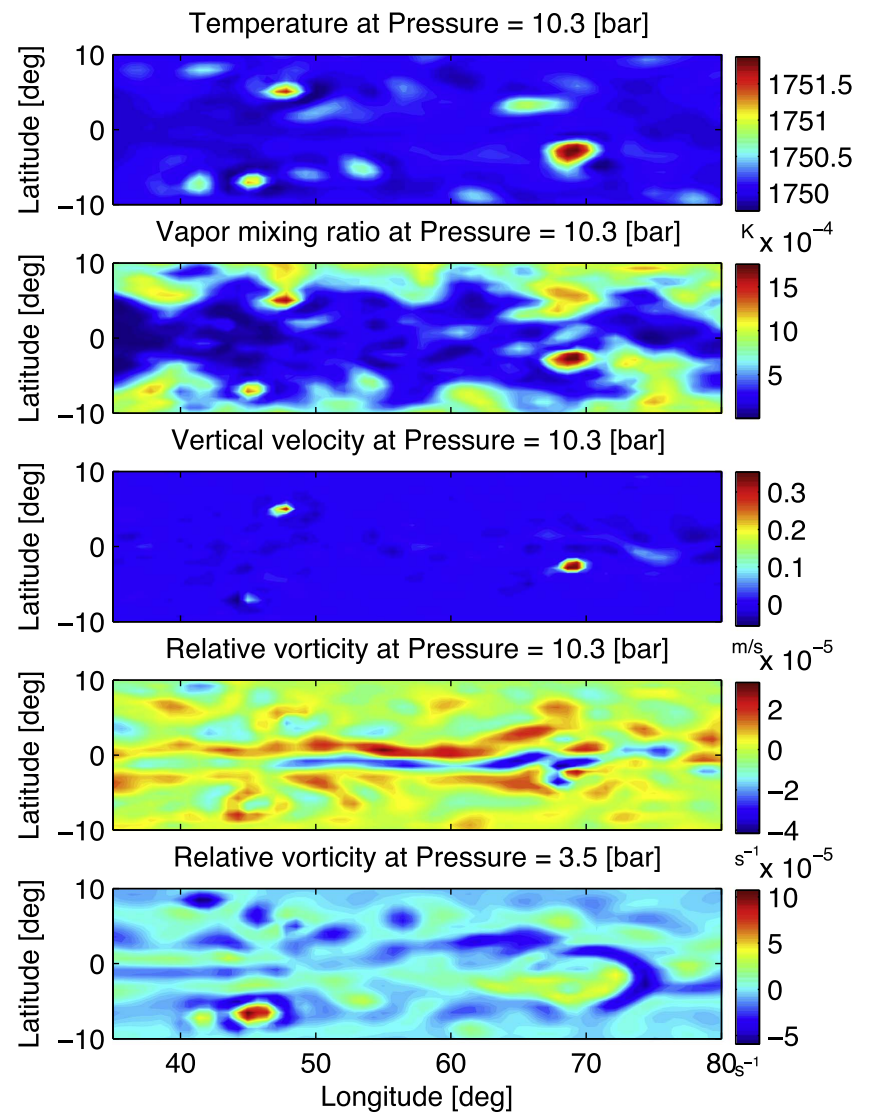

Figure 4. Snapshots of temperature, vapor mass mixing ratio, vertical velocity, and relative vorticity in a storm active region at about 1736 days for the nominal $\mathrm{T}$ dwarf simulation. Red in the vertical velocity map represents an upward direction. The condensation level for this simulation is about 11 bars.

shown on the horizontal maps representing a local storm active area in Figure 4. The vertical relative vorticity $\zeta=\hat{k} \cdot \nabla \times v$ (lower two panels of Figure 4) measures the local spin of fluid in the horizontal direction. If $\zeta$ has the same sign as the Coriolis parameter $f$, the storm is cyclonic, whereas if $\zeta$ and $f$ have opposite signs, the storm is anticyclonic. In the northern hemisphere, the base of storms (near 9 bars) generally has positive $\zeta$, and the top of storms (near 3.5 bars) generally has negative $\zeta$. The dynamical picture for a single storm is that because of latent heating, the lower density of the storm column causes a greater vertical spacing of isobars, i.e., constant-pressure lines bow downward at the base of the storm and upward at the top of the storm. This causes a low-pressure center at the base of the storm and a high-pressure center at the top of the storm with respect to the surrounding environmental air at a given altitude. As a result, horizontal flow converges and diverges due to pressure gradient forces at the base and top of the storm, respectively. Meanwhile, the flow is accelerated by the Coriolis force, which drives the flow to become cyclonic at the base of the storm and anticyclonic at the top.

\subsection{Zonal Jets}

Large-scale latent heating drives global atmospheric circulation and forms zonal jets in our simulations, as discussed in Section 2.4. The time-averaged zonal-mean zonal jet configurations from simulations with three different radiative timescales $\left(\tau_{\text {rad }}=10^{5}, 10^{6}\right.$, and $10^{7} \mathrm{~s}$, with other parameters the same as those of the typical $\mathrm{T}$ dwarf in Section 4.1) are shown
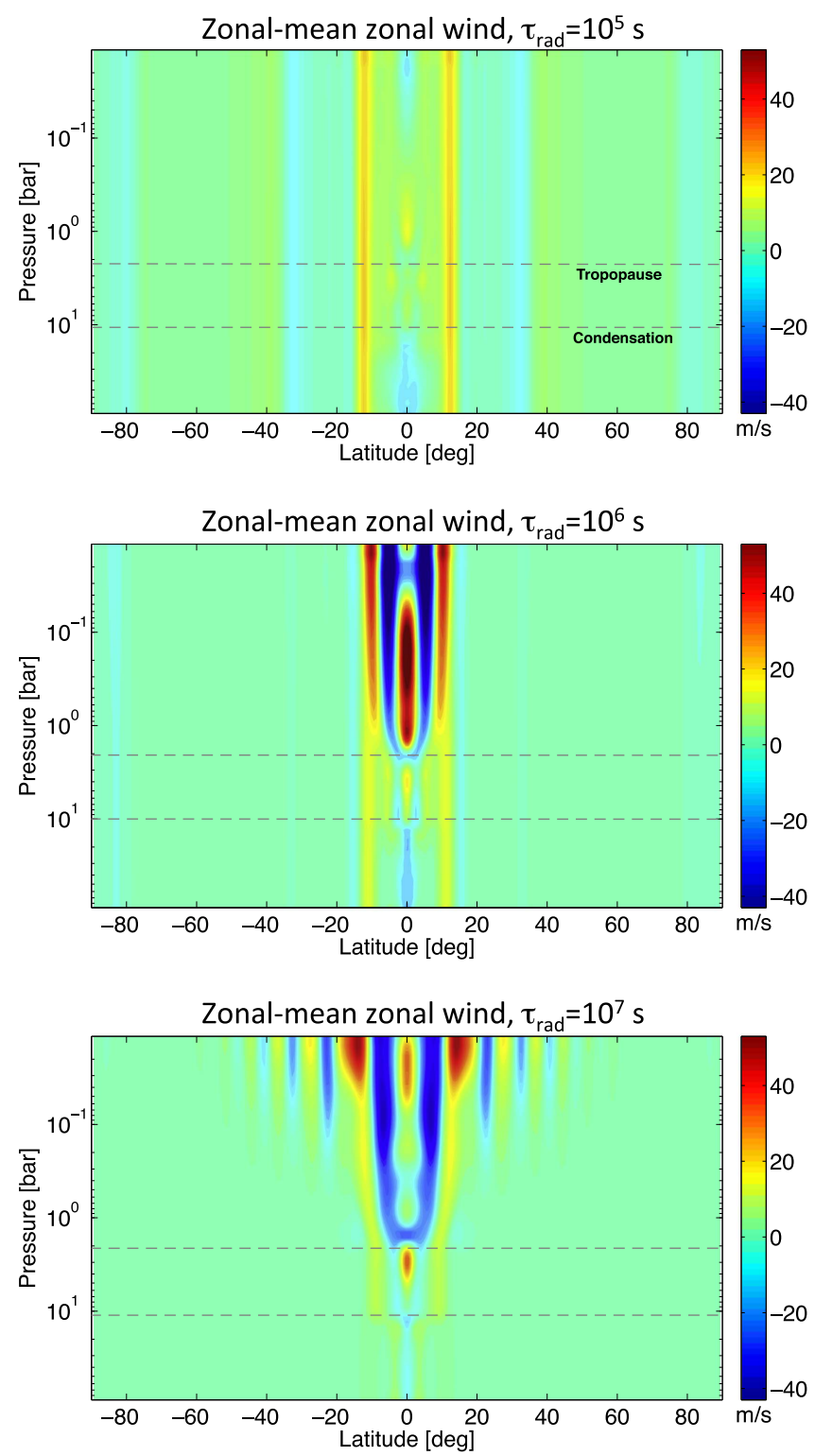

Figure 5. Time-averaged zonal-mean zonal wind as a function of latitude and pressure for three simulations with different radiative timescales $\tau_{\text {rad }}=10^{5} \mathrm{~s}$ (upper), $10^{6} \mathrm{~s}$ (middle), and $10^{7} \mathrm{~s}$ (lower). The dashed lines in each panel mark the pressure for the condensation level and the tropopause. The tropopause is here defined as the vertical level above which the lapse rate $d \ln T / d \ln p$ is less than 0.268 , which is slightly lower than the adiabatic lapse rate $R / c_{p}=0.2857$ but enough to stratify flows inside storms.

in Figure 5. The results are averaged over about 1000 days after the models are equilibrated. In general, two strong eastward subtropical jets form at $\pm 12^{\circ}$ latitudes, and weak jets form at mid-to-high latitudes, which are symmetric about the equator. Equatorial jets are generally found westward below the condensation level, and eastward equatorial jets appear just above the condensation level. The equatorial jet speed increases with height to the tropopause because of the baroclinic structure due to latent heating, with the jet strength depending on the radiative timescale. Here, baroclinic means that constant-density surfaces are not aligned with constant-pressure surfaces, whereas barotropic means that the two surfaces are aligned. The local maximum jet speed near the tropopause is caused by the strong dynamical perturbations from eddies generated at the top of storms. Jets below the condensation 
level are generally weak, and subtropical jets are presumably driven by the Coriolis force ${ }^{2}$ on the meridional circulation in the deep atmosphere that results from the circulation of the upper active layer (Haynes et al. 1991; Showman et al. 2006; Lian \& Showman 2008). The jets extend into the upper stably stratified atmosphere. The circulation above the tropopause probably emerges from the absorption, dissipation, and breaking of upward propagating waves that are generated at the tropopause (Showman \& Kaspi 2013). There have been extensive studies showing that mechanical, wave-induced forcing is the dominant driver of stratospheric circulation on Earth despite the existence of equator-to-pole thermal forcing (see, for example, reviews by Andrews et al. 1987; Haynes 2005). In the conditions of our simulated atmospheres, where an isotropic equilibrium thermal structure is imposed, wave-induced mechanical forcing should be responsible for the stratospheric circulation. In fact, we have observed upward propagating waves from levels perturbed by latent heating in our simulations, which support our hypothesis. The jet structure exhibits differences with different radiative timescales $\tau_{\text {rad }}$, as $\tau_{\text {rad }}$ can affect the rate at which the characteristic horizontal temperature differences and dynamical perturbations are damped. First, the short $\tau_{\text {rad }}$ model shows a nearly barotropic jet structure, whereas relatively long $\tau_{\text {rad }}$ models show a baroclinic structure. Second, the jet speed is generally higher for the relatively long $\tau_{\text {rad }}$ model, presumably because there is more time for jets to pump up before dynamical perturbations are damped out; this relation has been formulated in Showman \& Kaspi (2013) using the quasi-geostrophic theory. The jet structure within about 2-3 pressure scale heights of the upper boundary for the model with $\tau_{\mathrm{rad}}=10^{7} \mathrm{~s}$ (the lower panel in Figure 5; jets from $20^{\circ}$ to $50^{\circ}$ latitude) is likely affected by the upper boundary conditions. We have tested models with higher upper boundaries $\left(10^{-3}\right.$ bar and less), and the dynamics deeper than about 3 pressure scale heights from the upper boundary remain almost the same as in the original model. We conclude that despite the imperfection of numerics near the upper boundary for the model with $\tau_{\text {rad }}=10^{7} \mathrm{~s}$, the dynamics presented here for the atmosphere below about 0.1 bar are physical.

\subsection{Area Fraction of Storms}

The small area fraction of moist plumes is visually shown in Figures 3 and 4 for a typical $\mathrm{T}$ dwarf model, in which the discretized warm areas only occupy a small fraction of the area at low latitudes where storms are active. The individual storms are small, typically having a diameter of around $2^{\circ}$ (a length of about $1700 \mathrm{~km}$, or three grid cells) above the condensation level at around 10 bars in all our models. As shown in Figure 3, the storms slightly expand near the tropopause, where flows undergo stratification and expand laterally. Here we display a more quantitative measurement of the storm fraction in models with different radiative timescales $\tau_{\text {rad. }}$. The storms are defined roughly between the condensation level and the tropopause, and they should have both a saturated mixing ratio of vapor $\left(q \geqslant q_{s}\right)$ and an upwelling velocity. Using the upward vertical velocity as an indicator for storms is reasonable in our case because, as shown below, the upward vertical velocities inside storms are much higher than the descending velocities outside

\footnotetext{
2 Notice that because of the fast rotation, the Coriolis parameter $f$ has a large magnitude of about $10^{-4} \mathrm{~s}^{-1}$ even at a $10^{\circ}$ latitude.
}
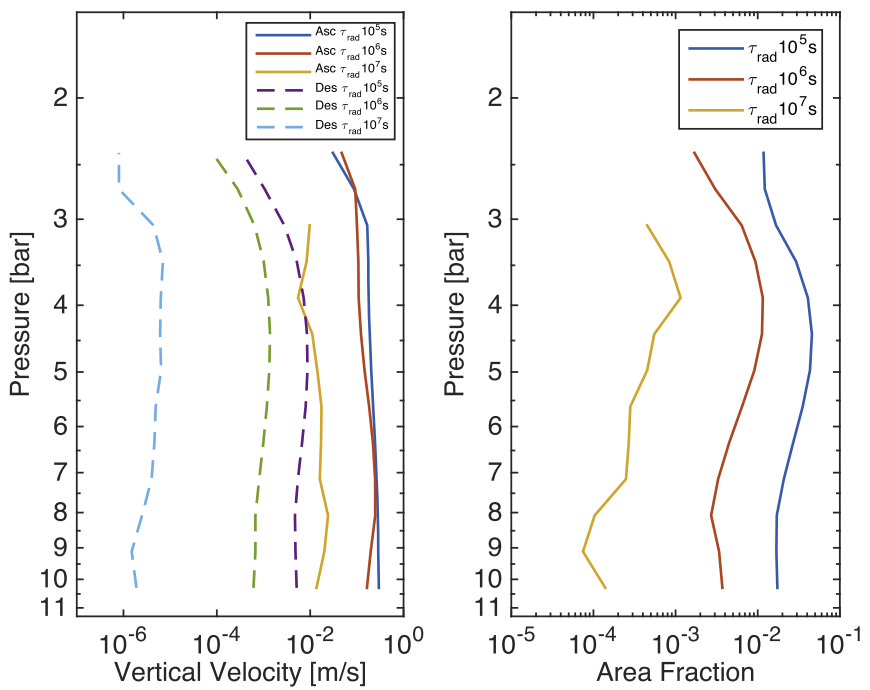

Figure 6. Left: absolute values for time-averaged ascending vertical velocity $\left(\mathrm{m} \mathrm{s}^{-1}\right)$ inside storms (solid lines) and descending velocity outside storms (dashed lines) as a function of pressure for models with $\tau_{\text {rad }}=10^{5}, 10^{6}$, and $10^{7} \mathrm{~s}$. Right: area fraction of storms $\sigma_{s}(p)$ as a function of pressure for the same models.

storms. We test the sensitivity of these criteria by choosing different numbers, for example, $q \geqslant 0.98 q_{s}$ or $q \geqslant 1.02 q_{s}$, and these different criteria do not affect the results. We define regions satisfying these two criteria as being inside storms. Regions not satisfying these criteria are defined as regions outside storms. We only count areas within $\pm 9^{\circ}$ latitudes since this is the primary region where storms occur. We first count vertical velocities as a function of pressure inside and outside storms using instantaneous snapshots of the vertical velocity field from simulations and then define the area fraction of storms $^{3}$ as $\sigma_{s}(p)=\left|\omega_{d}(p) / \omega_{a}(p)\right|$, where $\omega_{d}(p)$ and $\omega_{a}(p)$ are the spatially averaged vertical velocity outside storms and inside storms, respectively. Finally, $\sigma_{s}(p)$ is averaged over many snapshots at different simulation times over about 1000 days after the simulation equilibrates. The results are shown in Figure 6 as a function of pressure for models with $\tau_{\text {rad }}=10^{5}$, $10^{6}$, and $10^{7} \mathrm{~s}$. On the left panel, the vertical velocity is in modified $\log$-pressure coordinates $-H d(\ln p) / d t$, where $H$ is the pressure scale height; this velocity is approximately equal to the vertical velocity in height coordinates. This is a standard way of representing vertical velocity in pressure coordinates (e.g., Andrews et al. 1987). Physically, the quantity $d(\ln p) / d t$ is the vertical velocity expressed in units of scale heights per second, with positive being downward. Multiplying by $-H$ converts this to the vertical velocity in $\mathrm{m} \mathrm{s}^{-1}$, with positive being upward. As long as the structure of isobars does not change rapidly with respect to $z$ over time, this quantity is approximately equal to the vertical velocity in height coordinates. The magnitude of descending vertical velocities clearly decreases by order of magnitude with increasing $\tau_{\text {rad }}$. The ascending velocities are similar for $\tau_{\text {rad }}=10^{5}$ and $\tau_{\text {rad }}=10^{6} \mathrm{~s}$, but they are a factor of $\sim 5$ smaller for $\tau_{\text {rad }}=10^{7} \mathrm{~s}$. As a result, the area fraction of storms decreases by orders of magnitude as $\tau_{\text {rad }}$ increases. We quantitatively explore the mechanism controlling the area fraction in Section 5.1.

\footnotetext{
3 This is a definition based on a continuity argument, consistent with the definition in Section 2.
} 


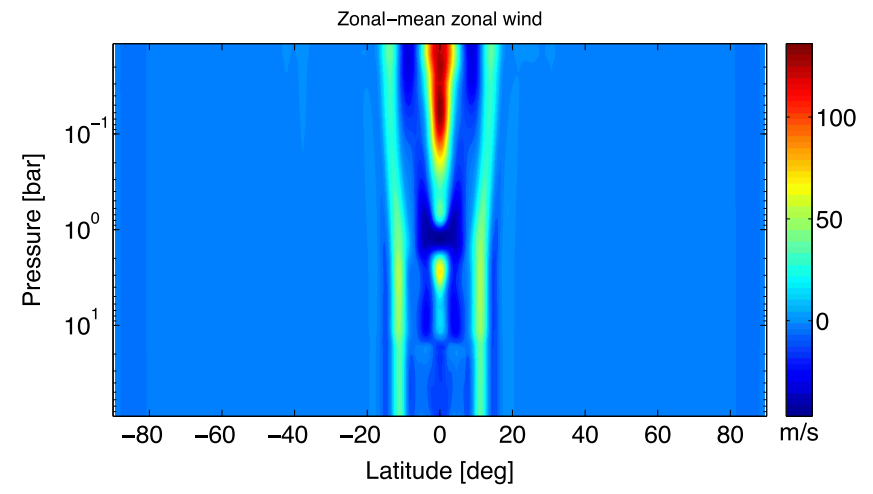

Figure 7. Time-averaged zonal-mean zonal wind for a $\mathrm{T}$ dwarf model with three times the solar abundance and $\tau_{\text {rad }}=10^{6} \mathrm{~s}$.

\subsection{Enhanced Abundance of Condensate}

Giant planets tend to have metal-rich atmospheres, having condensed out of the gas-depleted disks around preferentially metal-enriched host stars (Gonzalez 1997). In the context of our model, an enhanced metallicity means a greater abundance of silicate vapor, a higher latent heating, and thus a stronger atmospheric circulation. We have run models with three times the solar abundance of silicate vapor, which represent the possible conditions of directly imaged EGPs. Generally, the basic pattern of the condensation cycle and the zonal jet configuration are similar to the solar abundance models, but with larger temperature perturbations (proportional to the abundance of vapor), active storms occurring up to slightly higher latitudes, and higher wind speeds. Figure 7 shows the time-averaged zonal-mean zonal wind from a model with three times the solar abundance (typical for heavy-element abundances on Jupiter) and $\tau_{\text {rad }}=10^{6} \mathrm{~s}$. The zonal jet structure is very similar to that of our model with solar abundance (middle panel of Figure 5), except that the winds are enhanced by several factors.

\section{Discussion and Conclusions}

\subsection{What Controls the Area Fraction of Moist Plumes?}

Here we construct a simple model to quantitatively understand the area fraction of storms shown in Section 4.3 by constructing scaling relations for the governing Equations (3)(6). The physical picture of the model is comprised of statistically steady storms and subsidence outside the storms. At its top, the storm center has high pressure relative to the surroundings, which can drive an outward divergent flow; then the high-entropy air radiatively cools over time during the slow subsidence, reaching almost the same temperature as the environment at the condensation level by the requirement of a steady state. Here we ignore density variations due to the rainout of condensate. The area fraction of storms $\sigma_{s}$ is given by the requirement of continuity as

$$
\sigma_{s} \sim\left|\frac{\omega_{d}}{\omega_{a}}\right|
$$

where $\left|\omega_{d}\right| \ll\left|\omega_{a}\right|$. As presented in Section 4.1, storms mostly occur at low latitudes, where the Rossby number is large $(\gtrsim 1)$. Near the top of the storm, the horizontal force balance is primarily between the advection and pressure gradient forces. At low latitudes, the Coriolis force could still have a nontrivial magnitude compared to the advection force. Including the Coriolis force in our scaling induces only a mild correction to our final result (Equation (16)) and does not change our conclusion in this section. Therefore, for the sake of a clearer illustration of the physical mechanism controlling the fractional area of storms, using the force balance between advection and the pressure gradient is reasonable. Therefore, the balance in the horizontal momentum Equation (3) is $v \cdot \nabla_{p} v \sim-\nabla_{p} \Phi$, which, to order of magnitude, reads

$$
\frac{U^{2}}{\mathcal{L}} \sim \frac{\Delta \Phi}{\mathcal{L}}
$$

where $\Delta \Phi$ is the horizontal difference in gravitational potential between the top of the storm and its surroundings on a constant-pressure surface. From hydrostatic equilibrium (Equation (4)), we can estimate the difference between the pressure inside the storm and that outside by integrating over the column:

$$
\Delta \Phi \sim R \delta \ln p \Delta T
$$

where $\delta \ln p$ is the vertical difference in $\log$-pressure between the bottom and the top of the storm and $\Delta T$ is the characteristic difference between the horizontal temperature inside the storm and that outside. From the continuity Equation (5), the horizontal divergence at the storm given by $\nabla_{p} \cdot v \sim U / \mathcal{L}$ is balanced by the vertical divergence of ascent inside the storms. This implies

$$
\frac{U}{\mathcal{L}} \sim \frac{\omega_{a}}{\delta p},
$$

where $\delta p$ is the difference in pressure between the bottom and the top of the storm. Combining Equations (11)-(13) and assuming constant vertical velocity, we can estimate the ascending velocity $\omega_{a}$ by

$$
\omega_{a} \sim \frac{\delta p \sqrt{R \Delta T \delta \ln p}}{\mathcal{L}} .
$$

To estimate the descending velocity, we use the thermodynamic energy Equation (6) and assume that the vertical advection of the potential temperature is much larger than the horizontal advection. This is reasonable near the tropopause, where the vertical difference of potential temperature is much larger than the horizontal differences. We can then obtain a balance between radiative cooling and vertical advection, which, to order of magnitude, reads $\omega_{d} \frac{\delta \theta}{\delta p} \sim \frac{\theta-\theta_{\text {ref }}}{\tau_{\text {rad }}}$, where $\delta \theta$ is the vertical difference in potential temperature outside storms between pressure levels corresponding to the bottom and top of the storms. Imagining a thermodynamic loop where air rises in storms and subsides between storms, we expect that at the altitude of the storm top, the environmental air outside storms has just been detrained from the top of the storm and therefore that the potential temperatures of the storm air and environmental air are the same at the pressure of the storm top. Likewise, we expect that in a closed thermodynamic loop the potential temperatures of environmental and storm air are equal at the storm bottom. To close the system, we assume that in a global-mean and steady state, the higher-entropy air descending from the top of storms radiates away most of the entropy it 
gained from latent heating and relaxes to near the reference temperature when it reaches the bottom of the storms, implying $\delta \theta \sim \theta-\theta_{\text {ref }}$. Assuming constant $\omega_{d}$, we can estimate the descending velocity as

$$
\omega_{d} \sim \frac{\delta p}{\tau_{\text {rad }}} .
$$

This equation simply states that the rate of descent is bottlenecked by the efficiency of radiation: in order for the air outside storms to descend over the vertical height of a storm, the air has to lose entropy (since the environment is stratified), and thus this descent must occur on timescales comparable to the radiative time constant. Finally, the area fraction can be obtained by substituting $\omega_{d}$ and $\omega_{a}$ into Equation (10):

$$
\sigma_{s} \sim \frac{\mathcal{L}}{\tau_{\text {rad }} \sqrt{R \Delta T \delta \ln p}}
$$

This is essentially a timescale comparison, where $\mathcal{L} / \sqrt{R \Delta T \delta \ln p}$ is the dynamical ascent timescale driven by CAPE and $\tau_{\text {rad }}$ is the timescale driven by radiative dissipation. According to the results in Section 4 , taking $\mathcal{L} \sim 10^{6} \mathrm{~m}$, $\Delta T \sim 2.5 \mathrm{~K}, \delta \ln p \sim 1.3$, and $\tau_{\text {rad }} \sim 10^{5}, 10^{6}$, and $10^{7} \mathrm{~s}$, we have area fractions $\sigma_{s} \sim 10^{-1}, 10^{-2}$, and $10^{-3}$, respectively. Compared to the simulations on the right panel of Figure 6, our analytical model, to order of magnitude, agrees well with the maximum area fraction for different $\tau_{\text {rad }}$. The area fractions from our numerical results show variation as a function of pressure, generally off by a few factors to a factor of 10 compared to those from our analytical model. Given the simplicity of the scaling theory, we can explain the order-ofmagnitude decrement of the area fraction with increasing $\tau_{\text {rad }}$, illustrating the important regulation of radiation on moist convection.

Real cloud formation exhibits many complexities not accounted for in this simple scaling theory. For example, the intertropical convergence zone in Earth's tropical region shows organized regions of vigorous cumulus convection, containing transient cloud clusters rather than simply regions of steadystate precipitation and mean updrafts. This is a result of interactions between local cumulus convection and large-scale atmospheric circulation (Holton \& Hakim 2012, Chapter 11). The large-scale latent heating scheme in our model represents not the small-scale cumulus convection but rather the hydrostatic interaction of storms with their surroundings. To understand the interactions between subgrid moist convection and large-scale flow, we need a better parameterization of moist convection in future studies. Also, radiative feedback by cloud particles can play an important role in the development of cumulus clouds. Our analysis here will be tested using more realistic models in future studies.

\subsection{Thermal Structure}

The thermal structure of the atmosphere can be affected directly by latent heating via its effect on temperature and indirectly by the molecular weight effect via introducing a stratification layer above the condensation level. The upper panel of Figure 8 shows the potential temperature $\theta$ as a function of pressure for air outside and inside of storms from
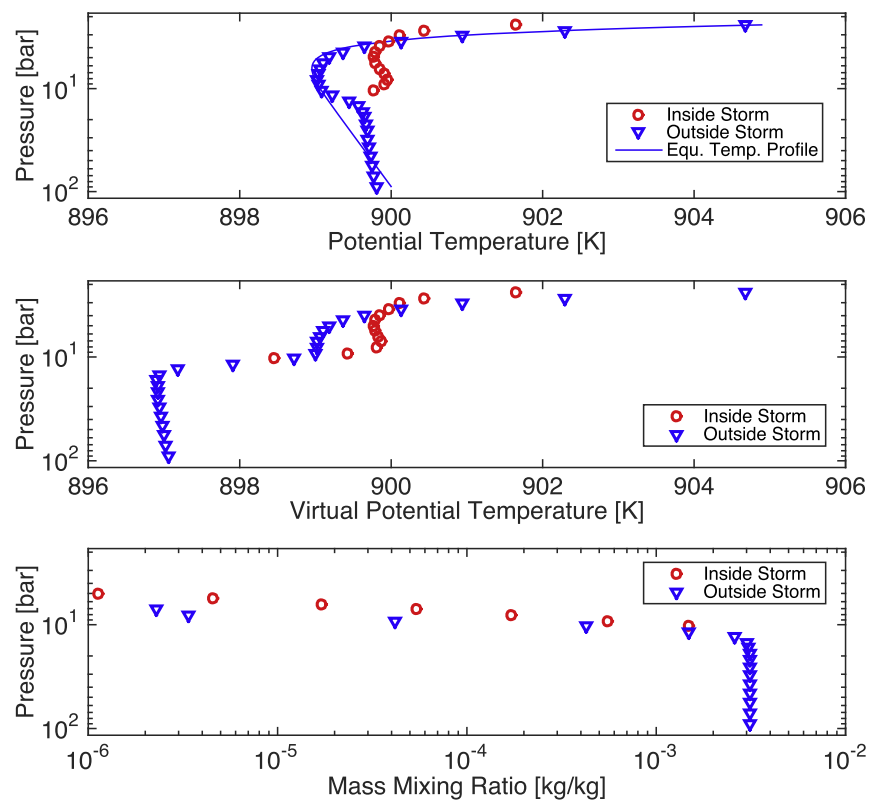

Figure 8. Thermal structure of the nominal simulation shown in Section 4 with radiative timescale $\tau_{\text {rad }}=10^{6} \mathrm{~s}$. The upper panel shows the potential temperature $\theta$ for air inside and outside storms as indicated by the labels as well as the equilibrium temperature profile. The middle panel shows the corresponding virtual potential temperature $\theta_{v}$ profile, and the lower panel shows the mixing ratio of condensable vapor for air outside and inside storms.

the nominal simulation with radiative timescale $\tau_{\text {rad }}=10^{6} \mathrm{~s}$ in Section 4 , and the middle panel shows the corresponding virtual potential temperature $\theta_{v}$ profile. The virtual potential temperature $\theta_{v}$ is defined as $\theta_{v}=\left(\frac{1+q / \epsilon}{1+q}\right) \theta$, where $\epsilon=m_{v} / m_{d}$ is the ratio of the molecular mass of condensable species to that of dry air and $q$ is the mass mixing ratio. It can be viewed as the theoretical potential temperature that a dry air parcel would have if the dry parcel had the same pressure and density as the moist air, so $\theta_{v}$ is a direct measurement of density. The solid line in the upper panel is the equilibrium background temperature profile prescribed by Equation (9). In the deep convective region, temperatures do not exactly follow the reference profile because dry motions tend to neutralize the thermal structure by having nearly constant $\theta$. However, due to the rainout of condensate, as shown in the lower panel of Figure 8, the layer just above the condensation level is stratified and is stable against dry convection. As a result, this thin layer just above the condensation level is not neutralized by dry motions. The stratification of this thin layer is better illustrated by the virtual potential temperature $\theta_{v}$ profile in the middle panel, in which $\theta_{v}$ increases with height despite the fact that $\theta$ actually decreases with height. Note that the increase of $\theta_{v}$ with height implies stratification against dry convection, accounting for both temperature and molecular weight gradients. The red circles represent (virtual) potential temperatures inside storms, and their profile is close to a moist adiabat. Interestingly, at the bottom of the stratosphere, "overshooting" of moist plumes occurs, in which the temperature inside storms (red circles) is lower than that of the surroundings. This suggests that ascending moist plumes penetrate into the stratosphere, inducing horizontal flow divergence, as described in Section 4.1. Due to the dry subsidence, layers immediately above the condensation level outside storms are unsaturated, decreasing the mean molecular weight with decreasing 
pressure. This contributes to a strong stratified background environmental profile at pressures near the condensation level. Interestingly, it also results in a lower density of the background air than that of the moist upwelling plumes. It can be seen in the middle panel of Figure 8, where air inside storms (red circles) has lower $\theta_{v}$ than background air (blue triangles) right above the condensation level. Overall, the molecular weight effect is likely to be important in regulating the way storms occur and the way they interact with their environment. This is similar to regional moist convection simulations in atmospheres of giant planets where water has greater molecular weight than hydrogen-helium mixtures (Nakajima et al. 2000; Sugiyama et al. 2014; Li \& Ingersoll 2015). Indeed, simulations from models without the molecular weight effect have more vigorous storm activity than those with the molecular weight effect. However, how this regulation works in a hydrostatic manner is unclear and needs more examination in future work.

\subsection{Implication for Observations}

Due to the lack of radiative transfer and cloud particles, we are unable to directly compare the simulated variability to the observed near-IR variability. Still, our results have important implications for observations. Storms driven by moist instabilities can extend vertically over several pressure scale heights and reach the photosphere. The vertical velocity inside largescale hydrostatic storms is high, and condensed particles can be lifted up to the storm top, forming cumulus clouds and inducing IR brightness variability. The storms can evolve on timescales of hours to days, and the cumulus clouds would be patchy due to spatially inhomogeneous moist convection and thus can help to explain the patchy clouds inferred in rapidly evolving near-IR light curves and observationally inferred surface maps of BDs. Recently, Karalidi et al. (2015) and Karalidi et al. (2016) have presented retrieval surface temperature maps for a few BDs based on near-IR light curves. Interestingly, the deduced temperature anomaly patterns are much larger than the expected Rossby deformation radius $\left(\sim 10^{7} \mathrm{~m}\right)$. This may be caused by a cluster of storms over a large fraction of the globe similar to that shown in Figure 4, which may produce a broad envelope of patchy clouds represented as a single large spot.

The $\mathrm{L} / \mathrm{T}$ transition occurs over a narrow range of effective temperatures accompanied by a $J$-band brightening (e.g., Allard et al. 2001; Burrows et al. 2006; Saumon \& Marley 2008), and its details remain poorly understood. Hypotheses include a change of sedimentation efficiency for condensates (Knapp et al. 2004) and the cloud deck's gradually becoming patchy as clouds form progressively deeper with increasing spectral type, allowing contributions from greater flux emitted from deeper levels (Ackerman \& Marley 2001; Burgasser et al. 2002; Marley et al. 2010). However, the detailed mechanisms for cloud breaking during the $\mathrm{L} / \mathrm{T}$ transition are yet unclear. Here we propose that the area fraction of moist convection can help to support the idea of cloud breaking during the $\mathrm{L} / \mathrm{T}$ transition. Moist convection occurs when high CAPE is available-that is, when the condensation level is much lower than the tropopause. This can also be quantified from Equation (16), where relatively high $\delta \ln p$ and a long radiative time constant $\tau_{\text {rad }}$ are needed to produce a small storm fraction. In the hotter L dwarfs, clouds first condense close to the upper stratified atmosphere (e.g., Tsuji 2002; Burrows et al. 2006), so moist convection cannot happen, and cloud morphology may be dominated by stratus clouds formed by more gradual processes, such as transport by waves (Freytag et al. 2010) or large-scale atmospheric flow (Showman \& Kaspi 2013). For the cooler dwarfs near the L/T transition, the condensation level gradually sinks below the tropopause; moist convection can thus occur with increasing CAPE, producing patchy cumulus clouds. Also, as the condensation level moves to a higher pressure, the radiative timescale $\tau_{\text {rad}}$, which can be approximated by $\tau_{\text {rad }} \sim \frac{p}{g} \frac{c_{p}}{4 \sigma T^{3}}$ (where $\sigma$ is the Stefan-Boltzmann constant), may become larger. According to our results, larger $\tau_{\text {rad }}$ can also decrease the storm area fraction. The changing of cloud patchiness during the $\mathrm{L} / \mathrm{T}$ transition can be a natural consequence of the change of CAPE and radiative timescale with increasing spectral type. We predict that if latent heating dominates cloud formation processes in the atmospheres of BDs and directly imaged EGPs, the fractional coverage area of clouds gets smaller as the spectral type goes through the $\mathrm{L} / \mathrm{T}$ transition from high to lower effective temperature. More realistic models are needed to test our hypothesis.

\subsection{Summary}

Latent heating from the condensation of various chemical species in BD atmospheres is important for shaping the atmospheric circulation and influencing cloud patchiness. We have illustrated the dynamical mechanisms of latent heating using an idealized atmospheric circulation model that includes the condensation cycle of silicate vapor with the molecular weight effect accounted for. For typical $\mathrm{T}$ dwarf models, zonal jets can be driven by large-scale latent heating. Temperature maps show inhomogeneous storm patterns that evolve on timescales of hours to days and can extend vertically over a pressure scale height or more to the tropopause. The fractional area of BDs covered by active storms is small. Based on a simple analytic model, we quantitatively explain the fractional area of storms and predict its dependence on the radiative timescale and CAPE. Our results have important implications for the observed near-IR variability and cloud properties across the $\mathrm{L} / \mathrm{T}$ transition. More GCMs with realistic clouds and radiative transfer are needed for better investigation of global circulation.

We thank Xi Zhang for helpful discussion. This work was supported by NASA Headquarters under the NASA Earth and Space Science Fellowship Program and NSF grant AST 1313444 to APS.

\section{References}

Ackerman, A. S., \& Marley, M. S. 2001, ApJ, 556, 872

Adcroft, A., Campin, J.-M., Hill, C., \& Marshall, J. 2004, MWRv, 132, 2845

Allard, F., Hauschildt, P. H., Alexander, D. R., Tamanai, A., \& Schweitzer, A. 2001, ApJ, 556, 357

Andrews, D. G., Holton, J. R., \& Leovy, C. B. 1987, Middle Atmosphere Dynamics, Vol. 40 (New York: Academic)

Apai, D., Radigan, J., Buenzli, E., et al. 2013, ApJ, 768, 121

Artigau, É., Bouchard, S., Doyon, R., \& Lafrenière, D. 2009, ApJ, 701, 1534 Barcilon, A., \& Gierasch, P. 1970, JAtS, 27, 550

Barman, T. S., Macintosh, B., Konopacky, Q. M., \& Marois, C. 2011a, ApJ, 733, 65

Barman, T. S., Macintosh, B., Konopacky, Q. M., \& Marois, C. 2011b, ApJL, 735, L39

Biller, B. A., Vos, J., Bonavita, M., et al. 2015, ApJL, 813, L23

Buenzli, E., Apai, D., Radigan, J., Reid, I. N., \& Flateau, D. 2014, ApJ, 782, 77 Buenzli, E., Saumon, D., Marley, M. S., et al. 2015, ApJ, 798, 127

Burgasser, A. J., Marley, M. S., Ackerman, A. S., et al. 2002, ApJL, 571, L151 
Burrows, A., Sudarsky, D., \& Hubeny, I. 2006, ApJ, 640, 1063

Cooper, C. S., Sudarsky, D., Milsom, J. A., Lunine, J. I., \& Burrows, A. 2003, ApJ, 586, 1320

Crossfield, I. J. M., Biller, B., Schlieder, J. E., et al. 2014, Natur, 505, 654

Cushing, M. C., Hardegree-Ullman, K. K., Trucks, J. L., et al. 2016, ApJ, 823,152

Emanuel, K. A. 1994, Atmospheric Convection (Oxford: Oxford Univ. Press) Emanuel, K. A., \& Raymond, D. 1993, The Representation of Cumulus Convection in Numerical Models (Boston, MA: American Meteorological Society), 145

Fegley, B., Jr., \& Lodders, K. 1996, ApJL, 472, L37

Flasar, F., \& Gierasch, P. 1986, JAtS, 43, 2683

Freedman, R. S., Lustig-Yaeger, J., Fortney, J. J., et al. 2014, ApJS, 214, 25

Freytag, B., Allard, F., Ludwig, H.-G., Homeier, D., \& Steffen, M. 2010, A\&A, 513, A19

Gierasch, P. J. 1976, Icar, 29, 445

Gierasch, P. J., Ingersoll, A. P., Banfield, D., et al. 2000, Natur, 403, 628

Gonzalez, G. 1997, MNRAS, 285, 403

Guillot, T. 1995, Sci, 269, 1697

Haynes, P. 2005, AnRFM, 37, 263

Haynes, P., McIntyre, M., Shepherd, T., Marks, C., \& Shine, K. P. 1991, JAtS, 48,651

Helling, C., \& Casewell, S. 2014, A\&ARv, 22, 1

Hinz, P. M., Rodigas, T. J., Kenworthy, M. A., et al. 2010, ApJ, 716, 417

Holton, J. R., \& Hakim, G. J. 2012, An Introduction to Dynamic Meteorology, Vol. 88 (New York: Academic)

Hubeny, I., \& Burrows, A. 2007, ApJ, 669, 1248

Ingersoll, A. P., Gierasch, P. J., Banfield, D., Vasavada, A. R. \& Galileo Imaging Team 2000, Natur, 403, 630

Ingraham, P., Marley, M. S., Saumon, D., et al. 2014, ApJL, 794, L15

Karalidi, T., Apai, D., Marley, M. S., \& Buenzli, E. 2016, ApJ, 825, 90

Karalidi, T., Apai, D., Schneider, G., Hanson, J. R., \& Pasachoff, J. M. 2015, ApJ, 814, 65

Knapp, G., Leggett, S. K., Fan, X., et al. 2004, AJ, 127, 3553

Leggett, S. K., Burningham, B., Saumon, D., et al. 2010, ApJ, 710, 1627

Li, C., \& Ingersoll, A. P. 2015, NatGe, 8, 398

Lian, Y., \& Showman, A. P. 2008, Icar, 194, 597

Lian, Y., \& Showman, A. P. 2010, Icar, 207, 373

Liu, B., \& Showman, A. P. 2013, ApJ, 770, 42

Lunine, J. I., \& Hunten, D. M. 1987, Icar, 69, 566

Macintosh, B., Graham, J., Barman, T., et al. 2015, Sci, 350, 64

Magalhães, J. A., Seiff, A., \& Young, R. E. 2002, Icar, 158, 410
Marley, M. S., \& Robinson, T. D. 2015, ARA\&A, 53, 279

Marley, M. S., Saumon, D., Cushing, M., et al. 2012, ApJ, 754, 135

Marley, M. S., Saumon, D., \& Goldblatt, C. 2010, ApJL, 723, L117

Marley, M. S., Seager, S., Saumon, D., et al. 2002, ApJ, 568, 335

Metchev, S. A., Heinze, A., Apai, D., et al. 2015, ApJ, 799, 154

Morley, C. V., Marley, M. S., Fortney, J. J., et al. 2014, ApJ, 787, 78

Nakajima, K., Takehiro, S.-I., Ishiwatari, M., \& Hayashi, Y.-Y. 2000, GeoRL, 27, 3129

Oppenheimer, B. R., Baranec, C., Beichman, C., et al. 2013, ApJ, 768, 24

Radigan, J., Jayawardhana, R., Lafrenière, D., et al. 2012, ApJ, 750, 105

Radigan, J., Lafrenière, D., Jayawardhana, R., \& Artigau, E. 2014, ApJ, 793, 75

Reuter, D., Simon-Miller, A., Lunsford, A., et al. 2007, Sci, 318, 223

Rossow, W. B. 1978, Icar, 36, 1

Salby, M. L. 2012, Physics of the Atmosphere and Climate (Cambridge: Cambridge Univ. Press)

Saumon, D., \& Marley, M. S. 2008, ApJ, 689, 1327

Saumon, D., Marley, M. S., Cushing, M. C., et al. 2006, ApJ, 647, 552

Saumon, D., Marley, M. S., Leggett, S. K., et al. 2007, ApJ, 656, 1136

Showman, A. P., Fortney, J. J., Lian, Y., et al. 2009, ApJ, 699, 564

Showman, A. P., Gierasch, P. J., \& Lian, Y. 2006, Icar, 182, 513

Showman, A. P., \& Kaspi, Y. 2013, ApJ, 776, 85

Skemer, A. J., Marley, M. S., Hinz, P. M., et al. 2014, ApJ, 792, 17

Snellen, I. A. G., Brandl, B. R., de Kok, R. J., et al. 2014, Natur, 509, 63

Stephens, D. C., Leggett, S. K., Cushing, M. C., et al. 2009, ApJ, 702, 154

Sugiyama, K., Nakajima, K., Odaka, M., Kuramoto, K., \& Hayashi, Y.-Y. 2014, Icar, 229, 71

Tsuji, T. 2002, ApJ, 575, 264

Vallis, G. K. 2006, Atmospheric and Oceanic Fluid Dynamics: Fundamentals and Large-scale Circulation (Cambridge: Cambridge Univ. Press)

Vasavada, A. R., \& Showman, A. P. 2005, RPPh, 68, 1935

Visscher, C., Lodders, K., \& Fegley, B., Jr. 2010, ApJ, 716, 1060

Visscher, C., \& Moses, J. I. 2011, ApJ, 738, 72

Wagner, K., Apai, D., Kasper, M., et al. 2016, Sci, 353, 673

Williams, G. P. 2003, JAtS, 60, 1270

Wilson, P. A., Rajan, A., \& Patience, J. 2014, A\&A, 566, A111

Yang, H., Apai, D., Marley, M. S., et al. 2015, ApJL, 798, L13

Yang, H., Apai, D., Marley, M. S., et al. 2016, ApJ, 826, 8

Zahnle, K. J., \& Marley, M. S. 2014, ApJ, 797, 41

Zhang, X., \& Showman, A. P. 2014, ApJL, 788, L6

Zhou, Y., Apai, D., Schneider, G. H., Marley, M. S., \& Showman, A. P. 2016, ApJ, 818, 176 